\title{
Continuum Charge Excitations in High-Valence Transition-Metal Oxides Revealed by Resonant Inelastic X-ray Scattering
}

\author{
Atsushi Hariki, ${ }^{1}$ Mathias Winder, ${ }^{1}$ and Jan Kuneš ${ }^{1,2}$ \\ ${ }^{1}$ Institute for Solid State Physics, TU Wien, 1040 Vienna, Austria \\ ${ }^{2}$ Institute of Physics, Czech Academy of Sciences, Na Slovance 2, 182 21 Praha 8, Czechia
}

(Dated: September 14, 2018)

\begin{abstract}
We present a theoretical investigation of the origin of Raman-like and fluorescencelike (FL) features of resonant inelastic x-ray scattering (RIXS) spectra. Using a combination of local-density approximation + dynamical mean-field theory and a configuration interaction solver for Anderson impurity model, we calculate the $L$-edge RIXS and x-ray absorption spectra of high-valence transition-metal oxides $\mathrm{LaCuO}_{3}$ and $\mathrm{NaCuO}_{2}$. We analyze in detail the behavior of the FL feature and show how it is connected to the details of electronic and crystal structure. On the studied compounds we demonstrate how material details determine whether the electron-hole continuum can be excited in the $L$-edge RIXS process.
\end{abstract}

The excitation spectrum is a fundamental characteristic that determines properties of a physical system. While the excitations of weakly correlated electrons can be built out of elementary ones and therefore different spectroscopic experiments show the similar spectra, e.g., the same gap for charge, spin and optical excitations, correlated materials such as transition metal oxides (TMO) are different $[1,2]$. Here, the connection between various types of excitations is buried deep in the experimentally unobservable wave function and different techniques are necessary to probe specific excitations.

Following the remarkable improvements of energy resolution in the past decade resonant inelastic x-ray scattering (RIXS) became a popular tool to study diverse materials [3]. Its sensitivity to a range of two-particle excitations enables observation of low-energy spin, orbital and charge excitations $(\sim 100 \mathrm{meV})[4-7]$ together with high-energy excitations $(\sim 1-10 \mathrm{eV})$, such as atomic multiplets or charge-transfer (CT) excitations [8-10]. The versatility of RIXS is paid for complicated interpretation of the resonant spectra which requires theoretical modeling.

Two features have been observed in the $L$-edge RIXS $(2 p \rightarrow 3 d \rightarrow 2 p)$ of TMO when scanning the incident photon energy $\omega_{\text {in }}$ across the x-ray absorption spectra (XAS): a Raman-like (RL) signal with a constant energy loss $\omega_{\text {loss }}=\omega_{\text {in }}-\omega_{\text {out }}$ and a fluorescencelike (FL) signal with a constant emission energy $\omega_{\text {out }}$, i.e., a linear dependence of $\omega_{\text {loss }}$ on $\omega_{\text {in }}[3,9,11-14]$. The analysis of material specific behavior of these features shows a potential for addressing questions concerning itinerancy of charge carriers and localization of charge excitations [12, 14, 15]. While several experimentally motivated interpretations were put forward, unified description of the atomic-like $\mathrm{RL}$ and itinerant FL features poses a theoretical challenge $[16,17]$.

The $L$-edge RIXS spectrum of the CT Mott insulator $\mathrm{NiO}[8,9]$ exhibits a RL behavior at $\omega_{\text {in }}$ of the main absorption peak well separated from the FL feature at $\omega_{\text {in }}$ of the $\mathrm{CT}$ satellite. A different behavior was reported in the negative-CT compound $\mathrm{NdNiO}_{3}$, where Bisogni et al. [15] observed merging of the low- $\omega_{\text {loss }}$ RL and FL features in $\mathrm{Ni} L_{3}$-RIXS. Moreover, the details of low- $\omega_{\text {loss }}$ FL feature exhibit distinct temperature dependence connected to opening of charge gap at low temperatures. Bisogni et al. interpreted the low- $\omega_{\text {loss }} \mathrm{FL}$ feature as a signature of unbound particle-hole pairs in the RIXS final state. Zhou et al. [12] studied $L$-edge RIXS in $\mathrm{LaAlO}_{3} / \mathrm{SrTiO}_{3}$ heterostructures and interpreted the relative intensity of the FL feature as a measure of itinerant carrier concentration. This conclusion was recently questioned by Pfaff et al. [14] who suggested that either RL or FL signals reflect the nature of the intermediate state of the RIXS process.

The commonly used cluster model with the TM $3 d$ and the neighboring $\mathrm{O} 2 p$ orbitals misses the FL feature completely due the lack of continuum (delocalized) particlehole excitations. This is remedied by the Anderson impurity model (AIM); however, the use of simple ad hoc bath densities of states $[8-10,15]$ does not allow to capture the important material details. To overcome this limitation we employ the local-density approximation $(\mathrm{LDA})+$ dynamical mean-field theory (DMFT) $[18,19]$ approach. The AIM with material specific hybridization density is then extended to include the core orbitals [20]. This approach, which we recently applied to study nonlocal screening effects in $L$-edge x-ray photoemission spectroscopy (XPS) [20], is here extended to analyze RIXS.

In order to focus on the physics of RIXS excitations, we avoid the uncertainties brought about by structural phase transition in nickelates and choose two isoelectronic $\mathrm{Cu}^{3+}$ compounds $\mathrm{LaCuO}_{3}$ and $\mathrm{NaCuO}_{2}$ as model systems for high-valence TMO. Both share a tiny CT energy [21-25] leading to small or no gap $[21,23,24,26,27]$. As we show later the key difference between the two compounds is the lattice geometry with corner-sharing $\mathrm{CuO}_{6}$ octahedra in $\mathrm{LaCuO}_{3}$, but edge-sharing ones in $\mathrm{NaCuO}_{2}$; see Fig. 1 .

The calculation proceeds in two steps. First, a stan- 
(a)

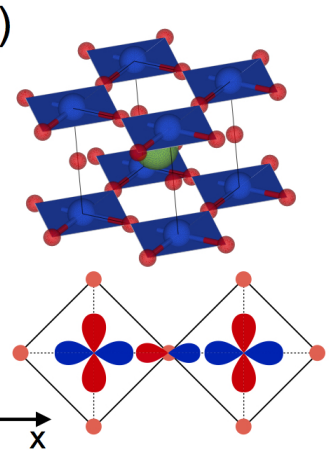

(b)

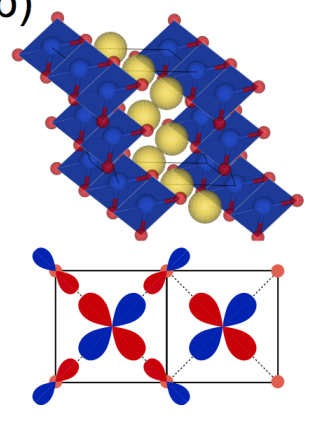

FIG. 1. The crystal structures of (a) $\mathrm{LaCuO}_{3}$ with $P 4 / m$ and and $C 2 / m$ space groups in paramagnetic and antiferromagnetic states, respectively [26] (b) $\mathrm{NaCuO}_{2}$ [28] visualized by VESTA [29]. The blue, red, green, and yellow circles represent $\mathrm{Cu}, \mathrm{O}, \mathrm{La}$ and $\mathrm{Na}$ atoms, respectively. The sketch of the $x y$ plane is shown together.

dard LDA+DMFT calculation is performed as follows. The LDA band structure obtained with Wien2K [30] is projected $[31,32]$ onto a $d p$ tight-binding model spanning the $\mathrm{Cu} 3 d$ and $\mathrm{O} 2 p$ orbitals and augmented with the electron-electron interaction within the $\mathrm{Cu} 3 d$ shell, giving the Hamiltonian

$$
H=\sum_{k}\left(\begin{array}{ll}
\boldsymbol{d}_{k}^{\dagger} & \boldsymbol{p}_{k}^{\dagger}
\end{array}\right)\left(\begin{array}{cc}
h_{k}^{d d}-\mu_{\mathrm{dc}} & h_{k}^{d p} \\
h_{k}^{p d} & h_{k}^{p p}
\end{array}\right)\left(\begin{array}{l}
\boldsymbol{d}_{k} \\
\boldsymbol{p}_{k}
\end{array}\right)+\sum_{i} W_{i}^{d d} .
$$

Here, $\boldsymbol{d}_{\boldsymbol{k}}\left(\boldsymbol{p}_{\boldsymbol{k}}\right)$ is an operator-valued vector whose elements are Fourier transforms of $d_{\alpha i}\left(p_{\gamma i}\right)$, that annihilate the $\mathrm{Cu}$ $3 d(\mathrm{O} 2 p)$ electron in the orbital $\alpha(\gamma)$ in the $i$ th unit cell. The on-site Coulomb interaction $W_{i}^{d d}$ is parametrized in the usual way $[33,34]$ with $U=7.5$ and $J=0.98 \mathrm{eV}$, typical for $\mathrm{Cu}$ systems [35]. The double-counting term $\mu_{\mathrm{dc}}$, which corrects for the $d-d$ interaction present in the LDA step, renormalizes the $p-d$ splitting and thus the CT energy. While several ad hoc schemes exist to compute $\mu_{\mathrm{dc}}$ (with somewhat different results), we treated $\mu_{\mathrm{dc}}$ as adjustable parameter fixed by comparison to the available $L$-edge XAS and valence XPS data, see the Supplementary Material (SM) [36]. The strong-coupling continuoustime quantum Monte Carlo method [37-39] with densitydensity approximation to the on-site interaction was used to solve the auxiliary AIM.

In the second step, we compute the RIXS spectra for AIM with DMFT hybridization density and $\mathrm{Cu} 2 p$ core states using the configuration interaction method [20]. The hybridization function, which encodes the information of how a given $\mathrm{Cu}$ orbital $\gamma$ exchanges electrons with the rest of the crystal, can be written as

$$
V_{\gamma}^{2}(\varepsilon)=\sum_{\alpha} \frac{V_{\alpha, \gamma}^{2}}{\varepsilon-\varepsilon_{\alpha, \gamma}}
$$

Here, $V_{\alpha, \gamma}$ is the hopping amplitude between the $\mathrm{Cu}$ ion and auxiliary orbitals at energies $\varepsilon_{\gamma, \alpha}$ [42], which rep-
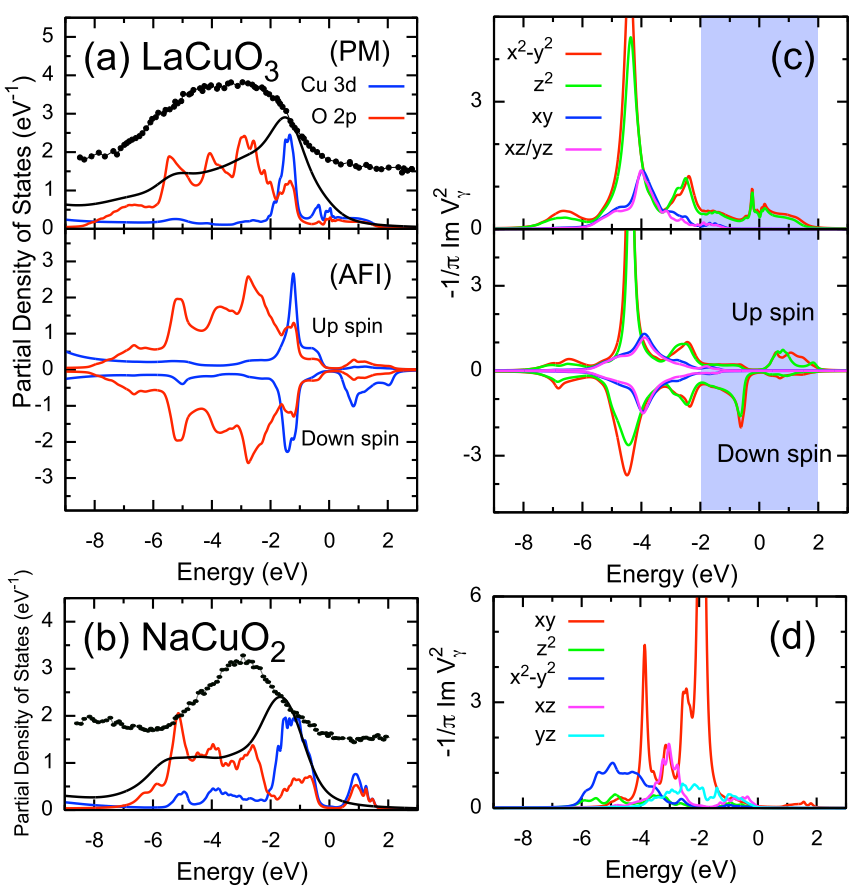

FIG. 2. LDA+DMFT $1 \mathrm{P}$ density of states for (a) $\mathrm{LaCuO}_{3}$ in the PM and AFI phases and (b) $\mathrm{NaCuO}_{2}$. The hybridization function $V_{\gamma}^{2}(\varepsilon)$ of $\mathrm{LaCuO}_{3}$ (c) in the PM and AFI phases and $\mathrm{NaCuO}_{2}$ (d). The energy origin is taken at $E_{F}$. The experimental XPS data (symbols) of $\mathrm{LaCuO}_{3}$ [23] and $\mathrm{NaCuO}_{2}[25]$ are compared to the theoretical ones (black line) [40]. $\mu_{\mathrm{dc}}=55.64 \mathrm{eV}$ is employed in the calculation.

resent the effect of the nearest-neighbor oxygen ligands as well as the more distant atoms [18, 20]. In practice, $V_{\gamma}^{2}(\varepsilon)$ obtained in the LDA+DMFT calculation is represented by 25 discretized bath states $\alpha$ for each $\mathrm{Cu}$ orbital $\gamma$ [20]. The RIXS intensity at finite temperature $T$ is given by $[9,10,43]$

$$
F_{\mathrm{RIXS}}\left(\omega_{\text {out }}, \omega_{\text {in }}\right)=\sum_{n} F_{\mathrm{RIXS}}^{(n)}\left(\omega_{\text {out }}, \omega_{\text {in }}\right) e^{-E_{n} / k_{B} T} / Z,
$$

where

$$
\begin{aligned}
F_{\text {RIXS }}^{(n)}\left(\omega_{\text {out }}, \omega_{\text {in }}\right) & =\sum_{f}\left|\sum_{m} \frac{\left\langle f\left|T_{\mathrm{e}}\right| m\right\rangle\left\langle m\left|T_{\mathrm{i}}\right| n\right\rangle}{\omega_{\text {in }}+E_{n}-E_{m}+i \Gamma_{\mathrm{L}}}\right|^{2} \\
& \times \delta\left(\omega_{\text {in }}+E_{n}-\omega_{\text {out }}-E_{f}\right) \\
& =\sum_{f}\left|\left\langle f\left|T_{\mathrm{e}} \frac{1}{\omega_{\text {in }}+E_{n}-H_{\text {imp }}+i \Gamma_{\mathrm{L}}} T_{\mathrm{i}}\right| n\right\rangle\right|^{2} \\
& \times \delta\left(\omega_{\text {in }}+E_{n}-\omega_{\text {out }}-E_{f}\right)
\end{aligned}
$$

Here, $|n\rangle,|m\rangle$, and $|f\rangle$ are the initial, intermediate and final states with energies $E_{n}, E_{m}$, and $E_{f}$, respectively, and $e^{-E_{n} / k_{B} T} / Z$ is the Boltzmann factor with the partition function $Z . \Gamma_{\mathrm{L}}$ is the lifetime width of the intermediate state, and $T_{\mathrm{i}}\left(T_{\mathrm{e}}\right)$ describes the dipole transition for 

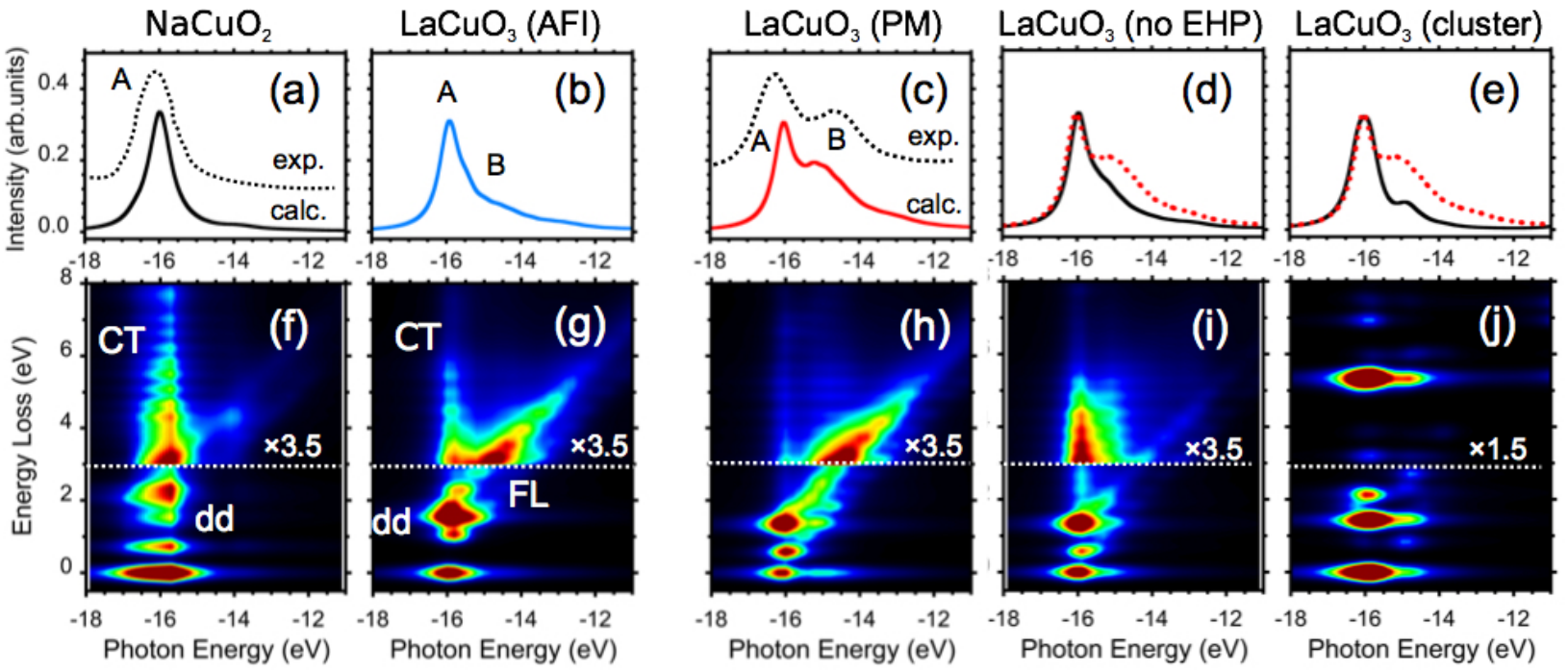

FIG. 3. The calculated $L_{3}$-edge XAS and RIXS spectra for (a),(f) $\mathrm{NaCuO}_{2}$ and $\mathrm{LaCuO}_{3}$ in (b),(g) AFI phase, (c),(h) PM phase, (d),(i) no EHP and (e),(j) $\mathrm{CuO}_{6}$ cluster model. The RIXS intensity with $\omega_{\text {loss }} \geq 3.0 \mathrm{eV}$ (horizontal dashed line) are magnified by 3.5 times $[1.5$ time for (e) $] . \mu_{\mathrm{dc}}=55.64 \mathrm{eV}$ is employed in the calculation. The LDA+DMFT spectrum for LaCuO 3 (PM) is shown by a dotted curve in (d) and (f), for comparison. The cluster-model results for $\mathrm{NaCuO}_{2}$ are found in $\mathrm{SM}$ [36]. The experimental XAS data (dashed line) for $\mathrm{LaCuO}_{3}(\mathrm{PM})$ and $\mathrm{NaCuO}_{2}$ are taken from Ref. [23] and Ref. [41]. The spectral broadening is considered using a Gaussian of $150 \mathrm{meV}$ for RIXS and a Lorentzian 300 meV for XAS (HWHM).

the incident (emitted) photon. $H_{\mathrm{imp}}$ is the AIM Hamiltonian augmented by the core orbitals and their interaction with $\mathrm{Cu} 3 d$ orbitals; see Eq. (3) in Ref. [20]. In the actual calculation the resolvent formulation on the second line of Eq. (1) is used. We also compute $L$-edge XAS spectra since the intermediate state $|m\rangle$ (corresponding to the final state of XAS) provides an important clue for interpretation of RIXS spectra. Details of the calculation can be found in SM [36].

In Figs. 2(a),2(b) we show the one-particle (1P) density of states of $\mathrm{LaCuO}_{3}$ and $\mathrm{NaCuO}_{2}$. The $\mu_{\mathrm{dc}}$ in the range of 55.64-57.64 eV yields results consistent with $\mathrm{Cu}$ $L$-edge XAS studies [23, 41, 44], see Fig. 3(a),3(c). The $\mu_{\mathrm{dc}}$ values provide also the best match with earlier valence XPS studies [23, 25] shown in Figs. 2(a),2(b). The deviations from the experimental XPS spectra may be due the uncertainty of the relative $\mathrm{Cu} 3 d: \mathrm{O} 2 p$ cross section and the surface sensitivity of XPS. In $\mathrm{NaCuO}_{2}$, in particular, the surface is prone to contamination leading to the appearance of $\mathrm{Cu}^{2+}$ ions $[41,45]$. Varying $\mu_{\mathrm{dc}}$ within the above range has only a minor impact on the RIXS spectra for both materials and does not affect our conclusions [36]. For $\mathrm{LaCuO}_{3}$, paramagnetic metal (PM) and antiferromagnetic insulator (AFI) solutions can be stabilized, similar to LDA $+U$ studies [24], indicating the Slater nature of the gap. Reflecting the unclear experimental situation [23, 24, 26, 27], we proceed with both states and use them later to demonstrate the effect of the small gap on RIXS. $\mathrm{NaCuO}_{2}\left(E_{\text {gap }} \approx 0.5 \mathrm{eV}\right)$ has a band-insulator character with a gap present already in the LDA solution $[45,46]$. Overall, the $1 \mathrm{P}$ density of states suggest existing phase space for continuum of the $p-p$ excitations in the few $\mathrm{eV}$ range. The calculated $\mathrm{Cu}$ $L_{3}$-edge XAS and RIXS spectra are shown in Fig. 3 . The XAS spectrum of $\mathrm{NaCuO}_{2}$ has a single-peak $(A)$, while that of $\mathrm{LaCuO}_{3}$ exhibits an additional shoulder $(B)$, enhanced in the PM phase, observed also in experiment for $\mathrm{NaCuO}_{2}$ [41] and $\mathrm{LaCuO}_{3}$ [23]. The shoulder $B$ is missing in the calculations on the $\mathrm{CuO}_{6}$ cluster model [23, 47], see Fig. 3(e), indicating that the shoulder is not of $d-d$ or $\mathrm{CT}$ origin but due to a final state delocalized beyond the $\mathrm{CuO}_{6}$ cluster. Such a nonlocal charge excitation is captured by the present approach [20].

The RIXS spectra of $\mathrm{NaCuO}_{2}$ and $\mathrm{LaCuO}_{3}$ are strikingly different. Tuning $\omega_{\text {in }}$ to the peak A of the XAS, two distinct $d-d$ transitions with RL behavior are found in both compounds, similar to another $\mathrm{Cu}^{3+}$ material $\mathrm{Zn}_{1-x} \mathrm{Cu}_{x} \mathrm{O}$ [48], followed by CT transitions with higher $\omega_{\text {loss }}$. However, at higher $\omega_{\text {in }}$ the RIXS of $\mathrm{LaCuO}_{3}$ yields a linear FL feature, with little difference between the AFI and PM phase. The FL feature is suppressed in $\mathrm{NaCuO}_{2}$ resembling the spectrum of the cluster model [36]. The calculated RIXS spectra of $\mathrm{LaCuO}_{3}$ reminds one of the experimental observation on $\mathrm{NdNiO}_{3}$ [15] with the FL feature starting at the $\omega_{\text {in }}$ on the $L_{3}$ main edge and not far above it as in $\mathrm{NiO}$. The continuum of unbound particle-hole pairs in the manner of Ref. [15] explains the FL feature in $\mathrm{LaCuO}_{3}$.

Why is the FL feature missing in $\mathrm{NaCuO}_{2}$ then? The small $\mathrm{NaCuO}_{2}$ gap cannot explain the absence of visible particle-hole excitations at $\omega_{\text {loss }}$ in the $3-4 \mathrm{eV}$ range. In fact, the experiment on $\mathrm{NdNiO}_{3}[15]$ and the calculations 

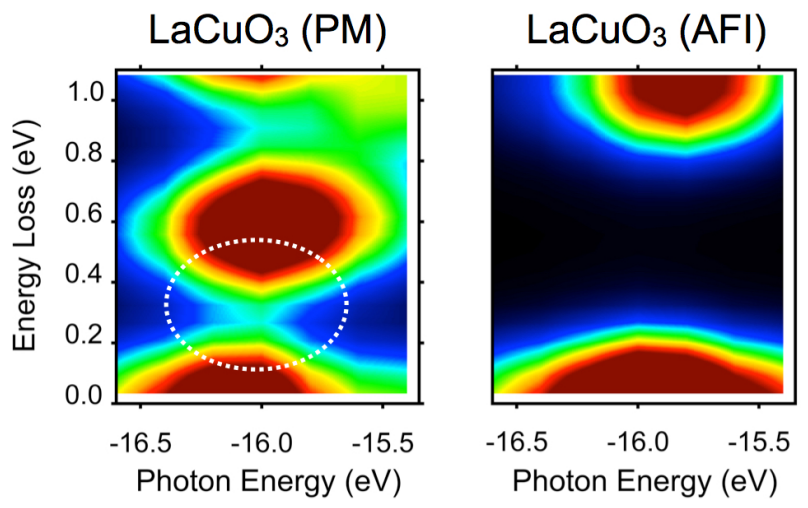

FIG. 4. Low $\omega_{\text {loss }}$ region $(<1.0 \mathrm{eV})$ of the RIXS map in the $\mathrm{PM}$ and AFI phases of $\mathrm{LaCuO}_{3}$. The FL feature continues to zero energy loss in the PM phase.

in the PM and AFI phases of $\mathrm{LaCuO}_{3}$ in Fig. 4 show that the gap opening affects the FL feature only at low $\omega_{\text {loss }}$. Moreover, the $\mathrm{NaCuO}_{2}$ 1P density of states, Fig. 2(b), exhibits noticeably higher density of states above and below the gap than $\mathrm{LaCuO}_{3}$, Fig. 2(a), suggesting a larger phase space for particle-hole excitations. To answer this question we show, in Figs. 2(c),2(d), the hybridization intensities $V_{\gamma}(\varepsilon)$. It is instructive to consider the $V_{\gamma}(\varepsilon)$ of the cluster model first [36]. Here, $V_{\gamma}(\varepsilon)$ is a single Dirac $\delta$ function peaked at the energy $\varepsilon_{p}$ of the ligand orbital, while the $\mathrm{O} 2 p$ density of states exhibits two peaks corresponding to the bonding and antibonding states.

A prominent peak in $V_{\gamma}(\varepsilon)$ (for the $e_{g}$ orbitals) due to hybridization to nearest-neighbor $\mathrm{O}$ ligands is found in both $\mathrm{LaCuO}_{3}$ and $\mathrm{NaCuO}_{2}$. The continuum part of $V_{\gamma}(\varepsilon)$ in the two materials reveals the difference. While in $\mathrm{LaCuO}_{3}$ a substantial hybridization intensity exists in the low-energy region of -2 to $2 \mathrm{eV}$ [blue shadow in Fig. 2(c)], the $V_{\gamma}(\varepsilon)$ of $\mathrm{NaCuO}_{2}$ resembles that of the cluster model with a weak continuum background. This is how the local quantity $V_{\gamma}(\varepsilon)$, relevant for description of the core-level excitation, encodes the information about bonding and lattice geometry. In $\mathrm{LaCuO}_{3}$, the cornersharing network of $\mathrm{CuO}_{6}$ octahedra allows electrons and holes to propagate through the strong $\mathrm{Cu}-\mathrm{O} \sigma$ bonds and thus gives rise to the continuum of $V_{\gamma}(\varepsilon)$. This is not possible in the chains of edge sharing $\mathrm{CuO}_{4}$ plaquettes in $\mathrm{NaCuO}_{2}$, where the neighboring $\mathrm{Cu}$ ions form $\sigma$ bonds with orthogonal O $2 p$ orbitals, see Fig. 1, and the crystal resembles a collection of weakly coupled $\mathrm{CuO}_{4}$ clusters.

How does the hybridization intensity affect the RIXS spectra? In Eq. (1), all intermediate states accessible in the XAS process contribute to RIXS in principle. We estimate that the intermediate states with $\left|E_{m}-E_{n}-\omega_{\text {in }}\right| \lesssim \Gamma_{\mathrm{L}}$, which approximately conserve energy in the partial XAS process, dominate while those outside this range cancel approximately out due to the varying sign of the denominator. Such a claim cannot
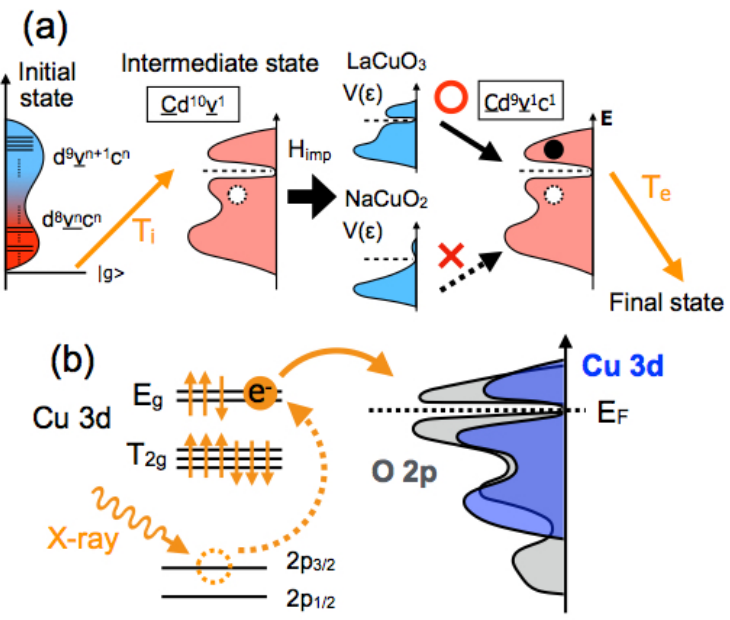

FIG. 5. Schematic of (a) electron-hole pair creation in the" RIXS process of $\mathrm{LaCuO}_{3}$ and $\mathrm{NaCuO}_{2}$, and (b) CT to the conduction states in the intermediate state.

be directly confirmed with the resolvent formulation of Eq. (1). It is, nevertheless, supported by the diagonal shape of the FL feature in the $\omega_{\text {in }}-\omega_{\text {loss }}$ plane, suggesting that a narrow range of intermediate states are "excited" that "decay" into a narrow range of final states with matching electron-hole excitation. The small hybridization intensity for $\varepsilon>0$ in $\mathrm{NaCuO}_{2}$ implies that (intermediate) states with different numbers of conduction electrons hybridize only weakly with one another. In $\mathrm{LaCuO}_{3}$ a RIXS process that we schematically write as $d^{8}+d^{9} \underline{v} \rightarrow \underline{C} d^{10} \underline{v}+\underline{C} d^{9} c \underline{v} \rightarrow d^{8} c \underline{v}$ ends up in a final state that can be characterized as the ground state plus an electron-hole pair in the continuum, see Fig 5(a), where $\underline{C}$, $\underline{v}$, and $c$ correspond to a hole in $2 p$ core level, in valence bands, and an electron in conduction bands, respectively. Such processes in $\mathrm{NaCuO}_{2}$ are strongly suppressed since states of the type $\underline{C} d^{10} \underline{v}$ and $\underline{C} d^{9} c \underline{v}$ hybridize only weakly. This is a local expression of the fact that in $\mathrm{NaCuO}_{2}$ a hole transferred from $\mathrm{Cu}$ to $\mathrm{O}$ has a small probability to escape the $\mathrm{CuO}_{4}$ cluster.

To test this interpretation, we switched off the hybridization to the conduction band in the intermediate states for the PM phase of $\mathrm{LaCuO}_{3}$; see Fig. 3(i). In practice, we have set $V_{\gamma}(\varepsilon>0)=0$ in the $H_{\text {imp }}$ of Eq. (1) while keeping $V_{\gamma}(\varepsilon)$ unrestricted in the initial and final states. In Fig. 5(b) the hybridization to the conduction band is sketched. The intensity of the FL feature is dramatically suppressed and the structure of the RIXS spectrum resembles that of $\mathrm{NaCuO}_{2}$. Although (unchanged) final states with excited electron-hole pairs exist, they cannot be resonantly excited by the RIXS process. This result supports the interpretation of the FL feature in Ti $L$-edge spectra by Pfaff et al. [14] and shows that the nature of hybridization in intermediate states is the dominant factor affecting the intensity of FL feature. Cutting hybridization to the conduction states 
affects also the XAS spectrum, Fig. 3(d), which loses the shoulder $B$ and overlaps with that of the cluster model, Fig. 3(e). This shows that intermediate states with localized and delocalized character coexist in this $\omega_{\text {in }}$ region, which leads to coexistence of FL and RL features in the RIXS spectrum.

In conclusion, we have studied the coexistence of RL and FL features in RIXS spectra of high-valence transition-metal oxides. We have shown how the IAM hybridization function encodes the information about the lattice environment and that it is the hybridization function rather than one-particle spectral density that determines the presence of FL feature. We predict that FL feature is present in the $L$-edge RIXS spectra of $\mathrm{LaCuO}_{3}$ both in the PM and AFM phases, while it is absent in the isoelectronic $\mathrm{NaCuO}_{2}$, experimental verification of which is highly desirable. We have interpreted this numerical observation in terms of crystal geometry. Comparing the RIXS spectra of PM and AFM $\mathrm{LaCuO}_{3}$, we have shown that the low- $\omega_{\text {loss }}$ details of the FL feature are sensitive to opening of a small gap similar to the experimental observation on $\mathrm{NdNiO}_{3}$ [15]. The present results show that the FL component of the RIXS spectra is rather material specific, and its interpretation requires advanced manybody calculations.

The authors thank R. Claessen, M. Sing, P. Scheiderer, T. Uozumi, R.-P. Wang, A. Sotnikov, and J. Fernández Afonso for fruitful discussions. The authors are supported by the European Research Council (ERC) under the European Union's Horizon 2020 research and innovation programme (Grant Agreement No. 646807EXMAG). The computational calculations were performed at the Vienna Scientific Cluster (VSC).

[1] M. Imada, A. Fujimori, and Y. Tokura, Rev. Mod. Phys. 70, 1039 (1998).

[2] D. I. Khomskii, Transition Metal Compounds (Cambridge University Press, Cambridge, England, 2014).

[3] L. J. P. Ament, M. van Veenendaal, T. P. Devereaux, J. P. Hill, and J. van den Brink, Rev. Mod. Phys. 83, 705 (2011).

[4] D. Betto, Y. Y. Peng, S. B. Porter, G. Berti, A. Calloni, G. Ghiringhelli, and N. B. Brookes, Phys. Rev. B 96, 020409 (2017).

[5] G. Fabbris, D. Meyers, L. Xu, V. M. Katukuri, L. Hozoi, X. Liu, Z.-Y. Chen, J. Okamoto, T. Schmitt, A. Uldry, B. Delley, G. D. Gu, D. Prabhakaran, A. T. Boothroyd, J. van den Brink, D. J. Huang, and M. P. M. Dean, Phys. Rev. Lett. 118, 156402 (2017).

[6] J. Kim, D. Casa, M. H. Upton, T. Gog, Y.-J. Kim, J. F. Mitchell, M. van Veenendaal, M. Daghofer, J. van den Brink, G. Khaliullin, and B. J. Kim, Phys. Rev. Lett. 108, 177003 (2012).

[7] L. Braicovich, L. J. P. Ament, V. Bisogni, F. Forte, C. Aruta, G. Balestrino, N. B. Brookes, G. M. De Luca, P. G. Medaglia, F. M. Granozio, M. Radovic, M. Sal- luzzo, J. van den Brink, and G. Ghiringhelli, Phys. Rev. Lett. 102, 167401 (2009).

[8] G. Ghiringhelli, M. Matsubara, C. Dallera, F. Fracassi, R. Gusmeroli, A. Piazzalunga, A. Tagliaferri, N. B. Brookes, A. Kotani, and L. Braicovich, J. Phys. Condens. Matter. 17, 5397 (2005).

[9] M. Matsubara, T. Uozumi, A. Kotani, and J. C. Parlebas, J. Phys. Soc. Jpn. 74, 2052 (2005).

[10] F. de Groot and A. Kotani, Core Level Spectroscopy of Solids (CRC Press, Boca Raton, FL, 2014).

[11] A. Kotani and S. Shin, Rev. Mod. Phys. 73, 203 (2001).

[12] K.-J. Zhou, M. Radovic, J. Schlappa, V. Strocov, R. Frison, J. Mesot, L. Patthey, and T. Schmitt, Phys. Rev. B 83, 201402 (2011).

[13] T. Schmitt, L.-C. Duda, M. Matsubara, M. Mattesini, M. Klemm, A. Augustsson, J.-H. Guo, T. Uozumi, S. Horn, R. Ahuja, A. Kotani, and J. Nordgren, Phys. Rev. B 69, 125103 (2004).

[14] F. Pfaff, H. Fujiwara, G. Berner, A. Yamasaki, H. Niwa, H. Kiuchi, A. Gloskovskii, W. Drube, J. Gabel, O. Kirilmaz, A. Sekiyama, J. Miyawaki, Y. Harada, S. Suga, M. Sing, and R. Claessen, Phys. Rev. B 97, 035110 (2018).

[15] V. Bisogni, S. Catalano, R. J. Green, M. Gibert, R. Scherwitzl, Y. Huang, V. N. Strocov, P. Zubko, S. Balandeh, J.-M. Triscone, G. Sawatzky, and T. Schmitt, Nat Commun. 7, 13017 (2016).

[16] J. Vinson, J. J. Rehr, J. J. Kas, and E. L. Shirley, Phys. Rev. B 83, 115106 (2011).

[17] K. Gilmore, J. Vinson, E. Shirley, D. Prendergast, C. Pemmaraju, J. Kas, F. Vila, and J. Rehr, Comput. Phys. Commun. 197, 109 (2015).

[18] G. Kotliar, S. Y. Savrasov, K. Haule, V. S. Oudovenko, O. Parcollet, and C. A. Marianetti, Rev. Mod. Phys. 78, 865 (2006).

[19] A. Georges, G. Kotliar, W. Krauth, and M. J. Rozenberg, Rev. Mod. Phys. 68, 13 (1996).

[20] A. Hariki, T. Uozumi, and J. Kuneš, Phys. Rev. B 96, 045111 (2017).

[21] T. Mizokawa, H. Namatame, A. Fujimori, K. Akeyama, H. Kondoh, H. Kuroda, and N. Kosugi, Phys. Rev. Lett. 67, 1638 (1991).

[22] D. Khomskii, eprint arXiv:cond-mat/0101164 (2001), cond-mat/0101164.

[23] T. Mizokawa, A. Fujimori, H. Namatame, Y. Takeda, and M. Takano, Phys. Rev. B 57, 9550 (1998).

[24] M. T. Czyżyk and G. A. Sawatzky, Phys. Rev. B 49, 14211 (1994).

[25] T. Mizokawa, A. Fujimori, H. Namatame, K. Akeyama, and N. Kosugi, Phys. Rev. B 49, 7193 (1994).

[26] J. F. Bringley, B. A. Scott, S. J. La Placa, T. R. McGuire, F. Mehran, M. W. McElfresh, and D. E. Cox, Phys. Rev. B 47, 15269 (1993).

[27] S. Darracq, S. Matar, and G. Demazeau, Solid State Commun. 85, 961 (1993).

[28] N. E. Brese, M. O'Keeffe, R. B. V. Dreele, and V. G. Young, J. Solid State Chem 83, 1 (1989).

[29] K. Momma and F. Izumi, J. Appl. Crystall. 44, 1272 (2011).

[30] P. Blaha, K. Schwarz, G. Madsen, D. Kvasnicka, and J. Luitz, WIEN2k, An Augmented Plane Wave + Local Orbitals Program for Calculating Crystal Properties (Karlheinz Schwarz, Techn. Universitat Wien, Austria, 2001, ISBN 3-9501031-1-2). 
[31] J. Kuněs, R. Arita, P. Wissgott, A. Toschi, H. Ikeda, and K. Held, Comput. Phys. Commun. 181, 1888 (2010).

[32] A. A. Mostofi, J. R. Yates, G. Pizzi, Y.-S. Lee, I. Souza, D. Vanderbilt, and N. Marzari, Comput. Phys. Commun. 185, 2309 (2014).

[33] E. Pavarini, E. Koch, A. Lichtenstein, and D. E. Vollhardt, The LDA+DMFT approach to strongly correlated materials, Schriften des Forschungszentrums Jülich : Modeling and Simulation, Vol. 1 (2011) record converted from VDB: 12.11.2012.

[34] E. Pavarini, "Electronic Structure Calculations with LDA+DMFT," in Many-Electron Approaches in Physics, Chemistry and Mathematics, Mathematical Physics Studies, ISBN 978-3-319-06378-2. Springer International Publishing Switzerland, 2014, p. 321, edited by V. Bach and L. Delle Site (2014) p. 321.

[35] V. I. Anisimov, J. Zaanen, and O. K. Andersen, Phys. Rev. B 44, 943 (1991).

[36] See Supplementary Material for details of the computation, double-counting correction dependence of XAS, RIXS and $1 \mathrm{P}$ density of states, and hybridization function, which includes Refs. [49-51].

[37] P. Werner, A. Comanac, L. de' Medici, M. Troyer, and A. J. Millis, Phys. Rev. Lett. 97, 076405 (2006).

[38] L. Boehnke, H. Hafermann, M. Ferrero, F. Lechermann, and O. Parcollet, Phys. Rev. B 84, 075145 (2011).

[39] H. Hafermann, K. R. Patton, and P. Werner, Phys. Rev. B 85, 205106 (2012).

[40] We used the same instrumental broadening and the relative $\mathrm{Cu} 3 d$ : O $2 p$ cross section as in Ref. [23], where the atomic cross section is taken from Ref. [52].
[41] D. D. Sarma, O. Strebel, C. T. Simmons, U. Neukirch, G. Kaindl, R. Hoppe, and H. P. Müller, Phys. Rev. B 37, 9784 (1988).

[42] In both materials an independent set of $\alpha$-orbitals can be, to a good approximation, chosen for each $\mathrm{Cu}$ orbital.

[43] H. A. Kramers and W. Heisenberg, Z. Phys. 31, 681 (1925)

[44] A. Webb, K. Kim, and C. Bouldin, Solid State Commn. 79, 507 (1991).

[45] D. Choudhury, P. Rivero, D. Meyers, X. Liu, Y. Cao, S. Middey, M. J. Whitaker, S. Barraza-Lopez, J. W. Freeland, M. Greenblatt, and J. Chakhalian, Phys. Rev. B 92, 201108 (2015).

[46] D. J. Singh, Phys. Rev. B 49, 1580 (1994).

[47] K. Okada and A. Kotani, J. Phys. Soc. Jpn 68, 666 (1999).

[48] P. Thakur, V. Bisogni, J. C. Cezar, N. B. Brookes, G. Ghiringhelli, S. Gautam, K. H. Chae, M. Subramanian, R. Jayavel, and K. Asokan, J. Appl. Phys. 107, 103915 (2010).

[49] R. D. Cowan, The Theory of Atomic Structure and Spectra (Los Alamos Series in Basic and Applied Sciences) (University of California Press, 1981).

[50] J. Sugar, Phys. Rev. B 5, 1785 (1972).

[51] D. Alders, L. H. Tjeng, F. C. Voogt, T. Hibma, G. A. Sawatzky, C. T. Chen, J. Vogel, M. Sacchi, and S. Iacobucci, Phys. Rev. B 57, 11623 (1998).

[52] J. Scofield, J. Electron. Spectrosc. Relat. Phenom. 8, 129 (1976). 


\title{
Supplementary material of "Continuum Charge Excitations in High-Valence Transition-Metal Oxides Revealed by Resonant Inelastic X-ray Scattering”
}

\author{
Atsushi Hariki, ${ }^{1}$ Mathias Winder, ${ }^{1}$ and Jan Kuneš ${ }^{1,2}$ \\ ${ }^{1}$ Institute for Solid State Physics, TU Wien, 1040 Vienna, Austria \\ ${ }^{2}$ Institute of Physics, Czech Academy of Sciences, Na Slovance 2, 18221 Praha 8, Czechia
}

\section{A. Computation of RIXS intensities}

$L$-edge RIXS cross-section $\frac{\delta^{2} \sigma}{\delta \Omega \delta \omega} \propto F_{\text {RIXS }}$ is described by the Kramers-Heisenberg formula [s1, s2],

$$
F_{\text {RIXS }}\left(\omega_{\text {out }}, \omega_{\text {in }}\right)=\sum_{n} F_{\text {RIXS }}^{(n)}\left(\omega_{\text {out }}, \omega_{\text {in }}\right) e^{-E_{n} / k_{B} T} / Z
$$

where

$$
\begin{aligned}
F_{\mathrm{RIXS}}^{(n)}\left(\omega_{\text {in }}, \omega_{\text {out }}\right) & =\sum_{f}\left|\sum_{m} \frac{\left\langle f\left|\hat{T}_{e}\right| m\right\rangle\left\langle m\left|\hat{T}_{i}\right| n\right\rangle}{\omega_{\text {in }}+E_{n}-E_{m}+i \Gamma_{\mathrm{L}}}\right|^{2} \delta\left(\omega_{\text {in }}+E_{n}-\omega_{\text {out }}-E_{f}\right) \\
& =\sum_{f}\left|\left\langle f\left|\hat{T}_{e} \frac{1}{\omega_{\text {in }}+E_{n}-\hat{H}+i \Gamma_{\mathrm{L}}} \hat{T}_{i}\right| n\right\rangle\right|^{2} \delta\left(\omega_{\text {in }}+E_{n}-\omega_{\text {out }}-E_{f}\right) .
\end{aligned}
$$

Here, $|n\rangle,|m\rangle$, and $|f\rangle$ represent the initial, intermediate, and final states with energies $E_{n}, E_{m}$, and $E_{f}$, respectively. $e^{-E_{n} / k_{B} T} / Z$ is the Boltzmann factor with the partition function $Z$. $\Gamma_{\mathrm{L}}$ is the lifetime width, and $\hat{T}_{i}\left(\hat{T}_{e}\right)$ is the transition operator that describes the x-ray absorption (emission) in the second-order optical process. In actual calculations, $\Gamma_{\mathrm{L}}$ is replaced by a constant value of $0.3 \mathrm{eV}$. The geometry setting including the x-ray polarization is encoded in the operators $\hat{T}_{i}$ and $\hat{T}_{e}$. In the present study, we employed the polarization configuration, where the polarization vector of $\mathrm{x}$-rays is perpendicular to the scattering plane [s3]. The incident (emitted) $\mathrm{x}$-ray has the energy $\omega_{\text {in }}\left(\omega_{\text {out }}\right)$, and energy loss is given by $\omega_{\text {loss }}=\omega_{\text {in }}-\omega_{\text {out }}$. The operator $\hat{H}$ is the Hamiltonian of the whole system and, in the LDA+DMFT approach, $\hat{H}$ is replaced by that of the Anderson impurity model (AIM) with the DMFT hybridization intensity [s4]. The AIM Hamiltonian $\hat{H}_{\mathrm{imp}}$ is augmented by the $2 p$ core orbitals and their interaction with $\mathrm{Cu} 3 d$ orbitals, and thus has the form

$$
\hat{H}_{\mathrm{imp}}=\hat{H}_{\mathrm{TM}}+\hat{H}_{\mathrm{hyb}} .
$$

The on-site Hamiltonian $\hat{H}_{\mathrm{TM}}$ is given as

$$
\hat{H}_{\mathrm{TM}}=\sum_{\gamma, \sigma} \tilde{\varepsilon}_{d}(\gamma) \hat{d}_{\gamma \sigma}^{\dagger} \hat{d}_{\gamma \sigma}+U_{d d} \sum_{\gamma \sigma>\gamma^{\prime} \sigma^{\prime}} \hat{d}_{\gamma \sigma}^{\dagger} \hat{d}_{\gamma \sigma} \hat{d}_{\gamma^{\prime} \sigma^{\prime}}^{\dagger} \hat{d}_{\gamma^{\prime} \sigma^{\prime}}-U_{d c} \sum_{\gamma, \sigma, \zeta, \eta} \hat{d}_{\gamma \sigma}^{\dagger} \hat{d}_{\gamma \sigma}\left(1-\hat{c}_{\zeta \eta}^{\dagger} \hat{c}_{\zeta \eta}\right)+\hat{H}_{\text {multiplet }},
$$

Here, $\hat{d}_{\gamma \sigma}^{\dagger}\left(\hat{d}_{\gamma \sigma}\right)$ and $\hat{c}_{\zeta \eta}^{\dagger}\left(\hat{c}_{\zeta \eta}\right)$ are the electron creation (annihilation) operators for $\mathrm{Cu} 3 d$ and $2 p$ electrons, respectively. The $\gamma(\zeta)$ and $\sigma(\eta)$ are the $\mathrm{Cu} 3 d(2 p)$ orbital and spin indices. The $\mathrm{Cu} 3 d$ site energies $\tilde{\varepsilon}_{d}(\gamma)=\varepsilon_{d}(\gamma)-\mu_{\mathrm{dc}}$ are the energies of the Wannier states $\varepsilon_{d}(\gamma)$ shifted by the double-counting correction $\mu_{\mathrm{dc}}$. The isotropic part of the $3 d-3 d\left(U_{d d}\right)$ and $2 p-3 d\left(U_{d c}\right)$ interactions are shown explicitly, while terms containing higher Slater integrals and the spin-orbit (SO) interaction are contained in $\hat{H}_{\text {multiplet. }}$ In the present study $U_{d c}=8.5 \mathrm{eV}$ is employed. The $\mathrm{SO}$ coupling within the $2 p$ shell and the anisotropic part of the $2 p$-3d interaction parameters $F^{k}, G^{k}$ are calculated with an atomic Hartree-Fock code. The $F^{k}$ and $G^{k}$ values are scaled down to $85 \%$ of their actual values to simulate the effect of intra atomic-configuration interaction from higher basis configurations neglected in the atomic calculation, which is a successful empirical treatment [s1, s3, s5, s6]. The $\hat{H}_{\text {hyb }}$ describes hybridization with the fermionic bath and is given as

$$
\hat{H}_{\mathrm{hyb}}=\sum_{\alpha, \gamma, \sigma} \epsilon_{\alpha, \gamma, \sigma} \hat{v}_{\alpha \gamma \sigma}^{\dagger} \hat{v}_{\alpha \gamma \sigma}+\sum_{\alpha, \gamma, \sigma} V_{\alpha, \gamma, \sigma}\left(\hat{d}_{\gamma \sigma}^{\dagger} \hat{v}_{\alpha \gamma \sigma}+\hat{v}_{\alpha \gamma \sigma}^{\dagger} \hat{d}_{\gamma \sigma}\right)
$$

The first term represents the energies of the auxiliary orbitals and the second term describes the hopping between the $\mathrm{Cu} 3 d$ ion and the auxiliary orbitals with the amplitude $V_{\alpha, \gamma, \sigma}$. Here $\hat{v}_{\alpha \gamma \sigma}^{\dagger}\left(\hat{v}_{\alpha \gamma \sigma}\right)$ is the creation (annihilation) operator for the auxiliary state with energy $\epsilon_{\alpha, \gamma, \sigma}$. The hybridization is assumed to be orbital (and spin) diagonal, which is a 
good approximation in the studied compounds. The configuration interaction scheme with 25 discretized-bath states representing the DMFT hybridization density is employed to compute RIXS intensities. In the discretization, we have employed uniform energy meshes in the intervals with sizable hybridization intensity (typically defined as larger than $5 \%$ of the peak value) [s4]. The initial state is computed using the Lanczos method. The intermediate state propagated by the resolvent as $\left(\omega_{\text {in }}+E_{n}-\hat{H}_{\mathrm{imp}}+i \Gamma_{\mathrm{L}}\right)^{-1} \hat{T}_{i}|n\rangle$ is computed using the conjugate-gradient method, and then its overlap to the final states are computed again using the Lanczos method.

(a) $\mathrm{LaCuO}_{3}(\mathrm{PM})$
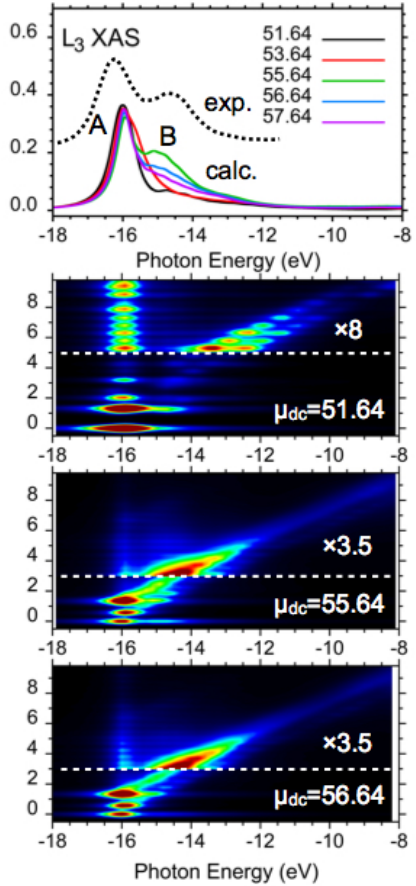

(b) $\mathrm{LaCuO}_{3}(\mathrm{AF})$

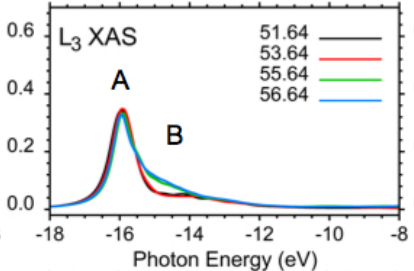

Photon Energy (eV)
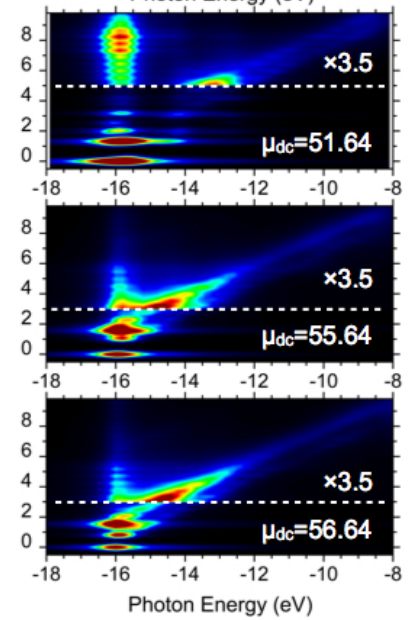

(c) $\mathrm{NaCuO}_{2}$

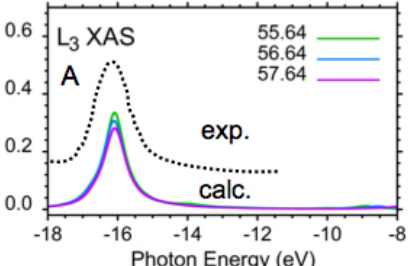

Photon Energy (eV)
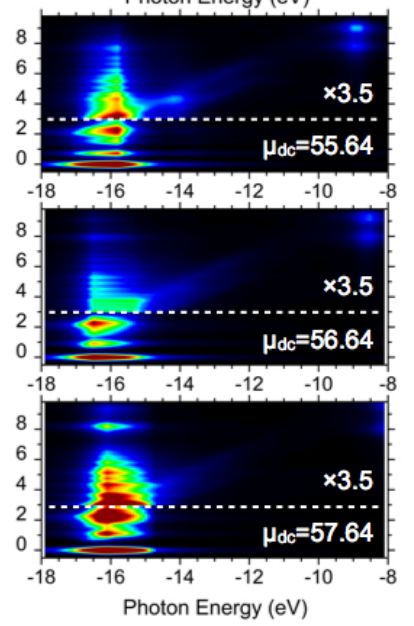

FIG. S.1. Calculated $L_{3}$-edge XAS and RIXS for (a) $\mathrm{LaCuO}_{3}(\mathrm{PM})$, (b) $\mathrm{LaCuO}_{3}(\mathrm{AF})$, and (c) $\mathrm{NaCuO}_{2}$. The experimental XAS data (dashed line) for $\mathrm{LaCuO}_{3}(\mathrm{PM})$ and $\mathrm{NaCuO}_{2}$ are taken from Ref. [s7] and Ref. [s8].

\section{B. Double-counting correction dependence of XAS, RIXS and valance spectra}

Figure S.1 shows $\mathrm{Cu} L$-edge XAS and RIXS calculated for different double-counting corrections $\mu_{\mathrm{dc}}$ in $\mathrm{LaCuO}_{3}$ and $\mathrm{NaCuO}_{2}$. The double-counting corrects for the $d-d$ interaction present in the LDA calculation. As one could find in the $d p$ Hamiltonian in the main text, $\mu_{\mathrm{dc}}$ renormalizes the $p-d$ splitting and a larger $\mu_{\mathrm{dc}}$ reduces the splitting, i.e. the charge-transfer (CT) energy. The XAS spectra of $\mathrm{LaCuO}_{3}$ with $\mu_{\mathrm{dc}}=51.64 \mathrm{eV}$, deep in the Mott insulating regime, shows a sharp main line $(\mathrm{A})$ and a weak multiplet feature around $-14 \sim-15 \mathrm{eV}$, resembling Ni $L$-edge XAS of NiO $\left(3 d^{8}\right.$ electronic configuration) [s9]. With $\mu_{\mathrm{dc}}$ increase (i.e., CT energy decrease), a new shoulder feature $B$ develops in both antiferromagnetic (AF) and paramagnetic $(\mathrm{PM})$ phases. The metal-insulator transition occurs at $\mu_{\mathrm{dc}} \sim 55.64 \mathrm{eV}$ in the PM solution, while a gap survives in the AF solution for $\mu_{\mathrm{dc}} \lesssim 56.74 \mathrm{eV}$. The $\mu_{\mathrm{dc}}=55.64-57.64 \mathrm{eV}$ yields a reasonable agreement to the experimental XAS data measured using the total electron yield method [s7]. The XAS of $\mathrm{NaCuO}_{2}$ shows a rather weak $\mu_{d c}$ dependence and does not show the shoulder $B$, consistent with the experimental observation [s8]. The estimated $\mu_{\mathrm{dc}}$ values give similar RIXS features in $\mathrm{LaCuO}_{3}$, showing coexisting fluorescencelike (FL) and Ramman-like features across the $\mathrm{Cu} L_{3}$ main line, while a small $\mu_{\mathrm{dc}}$, see $\mu_{\mathrm{dc}}=51.64 \mathrm{eV}$, gives a FL feature that appears far above the $L_{3}$ main line as in NiO [s10]. The calculated valance XPS with the estimated $\mu_{\mathrm{dc}}$ give a fair agreement to early photoemission data [s7, s11], see Fig. S.2. 
(a) $\mathrm{LaCuO}_{3}(\mathrm{PM})$

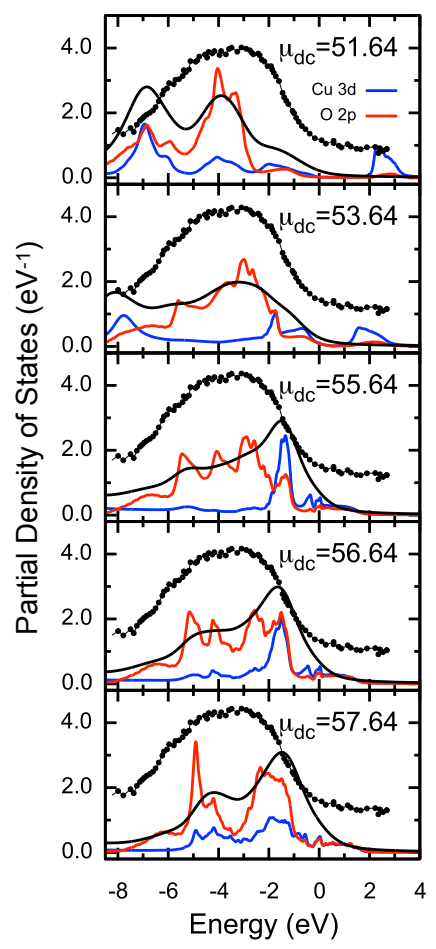

(b) $\mathrm{LaCuO}_{3}(\mathrm{AF})$

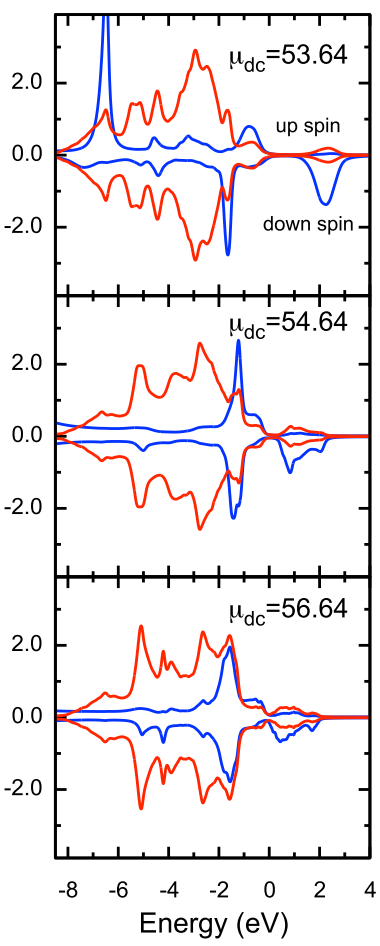

(c) $\mathrm{NaCuO}_{2}$

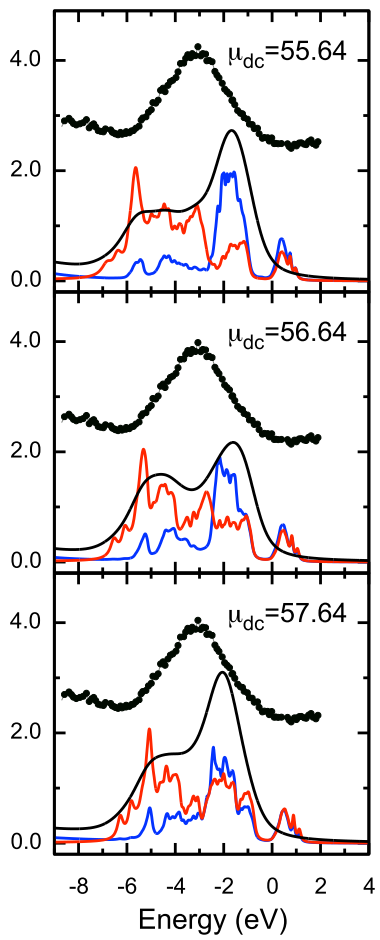

FIG. S.2. Calculated 1P density of states of (d) $\mathrm{LaCuO}_{3}$ (PM), (e) $\mathrm{LaCuO}_{3}$ (AF), and (f) $\mathrm{NaCuO}_{2}$ for different doublecounting corrections $\mu_{\mathrm{dc}}$. The calculated valence spectra (black line) are shown together. The experimental XPS data of $\mathrm{LaCuO}_{3}$ and $\mathrm{NaCuO}_{2}$ are taken from Ref [s7] and [s11], respectively.

\section{Hybridization density $V_{\gamma}^{2}(\varepsilon)$, XAS and RIXS spectra in cluster model}

Figure S.3 shows the hybridization density $V_{\gamma}^{2}(\varepsilon)$ of the studied compounds in the cluster model. Considering the crystal structure, see Fig. 1 in the manuscript, we here assumed $\mathrm{CuO}_{6}$ and $\mathrm{CuO}_{4}$ cluster for $\mathrm{LaCuO}_{3}$ and $\mathrm{NaCuO}_{2}$, respectively. The $V_{\gamma}^{2}(\varepsilon)$ shows a single-peak feature, as expected, while the energy position of the peaks depends on $\gamma$ because of the crystal-field splitting in $\mathrm{O} 2 p$ states.

Figure S.4 shows the $L_{3}$ XAS and RIXS spectra computed by the LDA+DMFT approach and the cluster model. For comparison, a magnified RIXS map of the RL features is shown for $\mathrm{LaCuO}_{3}$. We find a reasonable agreement in the energy positions of the RL features $(\lesssim 2.0 \mathrm{eV})$ between the two methods. However, the intensities and the resonance photon energies of the RL features differ between the two methods. These depend, in general, on the character of the intermediate states of the RIXS process. We remind that the cluster model does not describe the intermediate states of $\mathrm{LaCuO}_{3}$ properly, which leads to the absence of the shoulder $\mathrm{B}$ in the XAS spectrum of the cluster model (as compared to experiment).

[s1] F. de Groot and A. Kotani, Core Level Spectroscopy of Solids (CRC Press, 2014).

[s2] H. A. Kramers and W. Heisenberg, Zeitschrift für Physik 31, 681 (1925).

[s3] M. Matsubara, T. Uozumi, A. Kotani, and J. C. Parlebas, J. Phys. Soc. Jpn. 74, 2052 (2005).

[s4] A. Hariki, T. Uozumi, and J. Kuneš, Phys. Rev. B 96, 045111 (2017).

[s5] R. D. Cowan, The Theory of Atomic Structure and Spectra (Los Alamos Series in Basic and Applied Sciences) (University of California Press, 1981).

[s6] J. Sugar, Phys. Rev. B 5, 1785 (1972).

[s7] T. Mizokawa, A. Fujimori, H. Namatame, Y. Takeda, and M. Takano, Phys. Rev. B 57, 9550 (1998). 


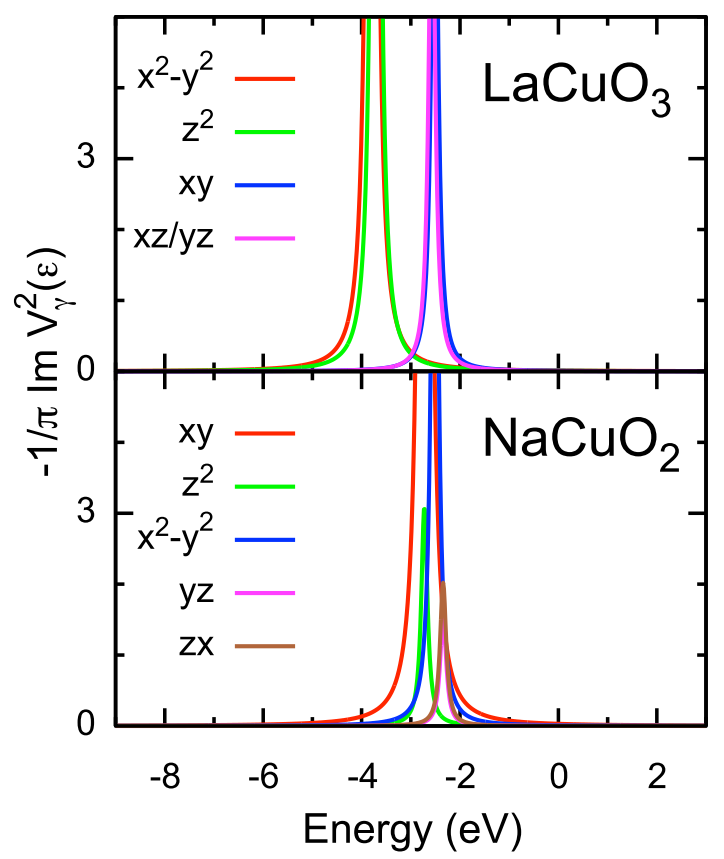

FIG. S.3. The hybridization function of $\mathrm{LaCuO}_{3}$ and $\mathrm{NaCuO}_{2}$ in the cluster model.
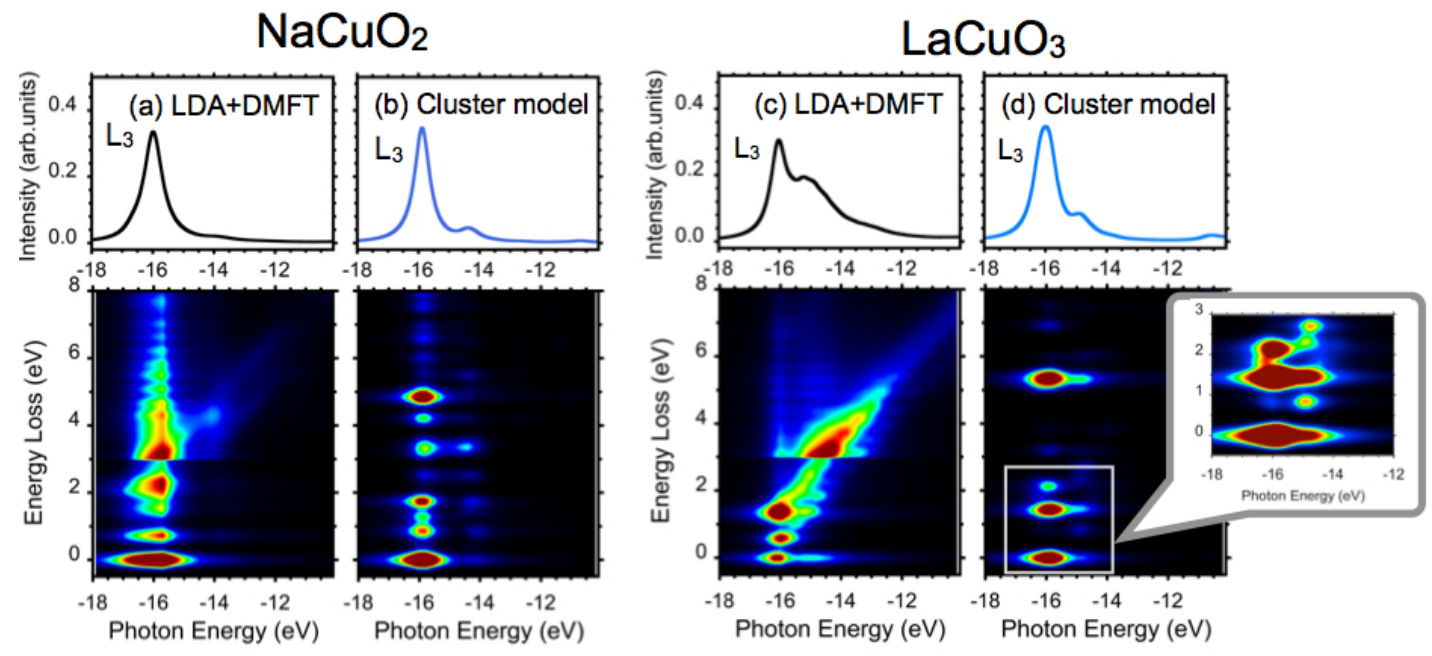

FIG. S.4. The $L_{3}$ XAS and RIXS spectra for $\mathrm{NaCuO}_{2}$ and $\mathrm{LaCuO}_{3}$ calculated by the LDA+DMFT approach and the cluster model. The magnification of the RL features in the cluster-model spectrum is shown, for comparison.

[s8] D. D. Sarma, O. Strebel, C. T. Simmons, U. Neukirch, G. Kaindl, R. Hoppe, and H. P. Müller, Phys. Rev. B 37, 9784 (1988).

[s9] D. Alders, L. H. Tjeng, F. C. Voogt, T. Hibma, G. A. Sawatzky, C. T. Chen, J. Vogel, M. Sacchi, and S. Iacobucci, Phys. Rev. B 57, 11623 (1998).

[s10] G. Ghiringhelli, M. Matsubara, C. Dallera, F. Fracassi, R. Gusmeroli, A. Piazzalunga, A. Tagliaferri, N. B. Brookes, A. Kotani, and L. Braicovich, J. Phys. Condens. Matter. 17, 5397 (2005).

[s11] T. Mizokawa, A. Fujimori, H. Namatame, K. Akeyama, and N. Kosugi, Phys. Rev. B 49, 7193 (1994). 